\title{
Absolute and convective instabilities in open shear layers
}

\section{Magnetohydrodynamic equilibrium}

\author{
M. Terra-Homem and R. Erdélyi
}

\begin{abstract}
Space and Atmosphere Research Centre, Dept. of Applied Mathematics, University of Sheffield, Hicks Building, Hounsfield Road, Sheffield S3 7RH, UK

e-mail: m.homem@sheffield.ac.uk; robertus@sheffield.ac.uk
\end{abstract}

Received 4 April 2003 / Accepted 18 September 2003

\begin{abstract}
The present paper is the continuation of our study of absolute and convective instabilities in open shear layers (Terra-Homem \& Erdélyi 2003). In this paper the effects of a magnetic field are included and a series of fully non-linear ideal polytropic 2D MHD numerical simulations is carried out. The amount of mean flow required to sweep away the perturbation before it grows and destroys the whole flow is calculated for various configurations of the magnetic field. The analytical results obtained by Fejér (1964) for a velocity discontinuity were recovered with a very good accuracy. The study focused on uniform, discontinuous and sheared magnetic fields. We found that the value of mean flow needed for a perturbation to become convectively unstable (critical mean flow) decreased with decreasing value of the plasma $\beta$. We also found that the low $\beta$ value side, of a discontinuous and sheared magnetic field, is predominant in the behaviour of the instability. Finally we show the thickness of the magnetic shear layer has no effect on the value of critical mean flow.
\end{abstract}

Key words. magnetohydrodynamics (MHD) - instabilities

\section{Introduction}

Shear flows are present in most solar-terrestrial applications and it is of fundamental importance to study their stability. There are many examples of such flows; for some specific examples please refer to the Introduction of the first paper of this study (Terra-Homem \& Erdélyi 2003).

There have only been a few studies of absolute and convective instabilities in astrophysical aspects. Wright et al. (2000) studied the convective nature of instabilities at the magnetospheric flanks. Mills et al. (2000) and Wright et al. (2002) studied a boundary with continuous velocity profile and a discontinuous magnetic field which was representative of the flank regions of the Earth's magnetopause. Ruderman et al. (2002) derived analytical solutions for the heliopause geometry and found that in the near flanks of the heliopause perturbations are absolutely stable but convectively unstable (see also the review by Ruderman 2000). Before, Baranov et al. (1992) studied the global behaviour of instabilities in the different sections of the heliopause boundary surface. Ruderman \& Fahr (1993) extended this study by including parallel magnetic fields in the interstellar side of the heliopause, and Ruderman \& Fahr (1995) studied the effect of magnetic fields in the solar wind side of the heliopause and found differences in the stability of the ecliptic and the polar parts of the heliopause discontinuity

Send offprint requests to: R. Erdélyi, e-mail: robertus@sheffield.ac.uk
(Ruderman et al. 1996). All of these studies have considered either a velocity discontinuity or a magnetic field discontinuity. For a more realistic configuration one has to consider that both the velocity and the magnetic field have a smooth transition. In the present paper we investigate differences in the behaviour of instabilities for three possible configurations of the magnetic field. First, we embed the plasma in a uniform magnetic field; next we allow for a discontinuous transition of the magnetic field; and finally we study the difference if the transition is a smooth function. This paper is a follow up of the study by Terra-Homem \& Erdélyi (2003) where a purely hydrodynamic case was considered. Including a magnetic field is important since most astrophysical plasmas are magnetic (e.g. the solar wind, magnetosphere, heliopause and solar structures).

In Sect. 2 the governing equations and the numerical method are presented. The different configurations of the initial value problem are also discussed. Section 3.1 shows the results of a test case study for a comparison between the numerical and previous analytical results. This section was motivated by the fact that it is known that a velocity discontinuity can be stabilised by the presence of uniform magnetic fields; the stability conditions for incompressible fluids have been derived (see Axford 1960, 1962). Later Fejér (1964) derived the stability conditions for a velocity discontinuity at a plane interface between two perfectly conducting, inviscid, compressible fluids, in the presence of uniform magnetic fields. This gives an analytical background to compare the numerical results with 
Table 1. The values of the parameters used in the simulations carried out in this work, and all the variables changed in each section.

\begin{tabular}{|c||c|c|c|c|}
\hline \hline \multicolumn{1}{|c||}{ fixed parameters } & \multicolumn{2}{c|}{$M_{\mathrm{s}}=0.83$} & \multicolumn{2}{c|}{$\gamma_{\mathrm{ad}}=1.66$} \\
\hline varied parameters & $h$ & $L$ & $\beta$ & $U_{0}$ \\
\hline \hline Section 3.1 & 0.1 & - & varied & 0.0 \\
\hline Section 3.2 & 2.0 & - & varied & varied \\
\hline Section 3.3.1 & 2.0 & 0.01 & varied & varied \\
\hline Section 3.3.2 & 2.0 & $\begin{array}{c}2.0 \\
4.0\end{array}$ & $\begin{array}{c}\beta_{1}=20 \\
\beta_{2}=10\end{array}$ & varied \\
\hline
\end{tabular}

previous established conditions. In Sect. 3.2 a study is carried out for the case of a velocity shear with a uniform magnetic field, and in Sect. 3.3 the case of a velocity shear with magnetic shear is investigated for a wide range of the parameters.

One should note that the interpolation in all the plots shown was always done with more than four data points. These were calculated in very time consuming numerical simulations and are represented by the various symbols in the plots (stars, triangles, squares, etc.). For that reason, the number of data points chosen was the minimum necessary for a correct interpolation of the respective fitting function. This choice has sacrificed accuracy in the results, but the purpose of the present article is to show the trend and effects of the different magnetic configurations. This was achieved in the plots presented here. When applying to more realistic configurations, one can then start considering a higher accuracy in the results. Also considering Figs. 8, 10 and 11, the interpolation is done with more data points than the ones seen in the plots. The reason why the data points are not all represented is that some lie below the $y$-axis (negative $\gamma$ ). In order to better identify the critical value of mean flow $\left(U_{\mathrm{c}}\right)$, it was chosen not to show the negative part of $y$ and so these points were excluded from the plot. The interpolation in these figures is a quadratic polynomial, and at least 5 data points were used, enough to represent adequately a second order polynomial. For the other figures geometric functions were used for the fitting.

This wide scope in the parameters allowed several different configurations to be studied. The mean velocity and the magnetic field intensity, as well as the width of the magnetic shear layer, were varied. Table 1 shows all the parameters used in the following results. Finally in Sect. 4 the most important results are summarized.

\section{Equilibrium configuration}

In this study the ideal polytropic MHD approximation has been used; this assumes that the change in the thermodynamical quantities of the plasma is a quasi-statical process and the equations read as follows:

$\partial_{\mathrm{t}} \rho+\nabla \cdot(\boldsymbol{v} \rho)=0$

$\partial_{\mathrm{t}}(\rho \boldsymbol{v})+\nabla \cdot(\boldsymbol{v} \rho \boldsymbol{v})-\nabla \cdot(\boldsymbol{B} \boldsymbol{B})+\nabla p_{\mathrm{tot}}=0$,

$\partial_{\mathrm{t}} \boldsymbol{B}+\nabla \cdot(\boldsymbol{v} \boldsymbol{B})-\nabla \cdot(\boldsymbol{B v})=0$,

$p_{\text {tot }}=p+\frac{B^{2}}{2}$

$p=c_{\mathrm{a}} \rho^{\gamma_{\mathrm{ad}}}$.
Here $\boldsymbol{v}$ is the velocity, $\boldsymbol{B}$ is the magnetic field, $\rho$ is the density, $p$ is the thermal pressure and $p_{\text {tot }}$ is the total pressure (magnetic plus thermal pressures). The magnetic field is measured in units for which the magnetic permeability is $1, \gamma_{\mathrm{ad}}=5 / 3$ (adiabatic index) and $c_{\mathrm{a}}$ is a constant related to the sound speed by

$c_{\mathrm{s}}=\sqrt{\frac{\gamma_{\mathrm{ad}} c_{\mathrm{a}} \rho^{\gamma_{\mathrm{ad}}}}{\rho}}$.

The condition $\nabla \cdot \boldsymbol{B}=0$ must be satisfied at all time. The equilibrium configuration follows the model used in the study of absolute and convective instabilities without the presence of magnetic field by Terra-Homem \& Erdélyi (2003). The velocity profile and the perturbation remain the same, i.e. a tanh profile is used for the horizontal component of the velocity and the perturbed vertical component of the velocity is a pulse with a Gaussian distribution in space. The spatial coordinates and velocity are normalised by half the thickness of the shear layer, and, by the difference of velocity in the two streams $\Delta U=$ $U_{1}-U_{2}$, respectively. The thickness of the velocity shear layer is fixed and is represented by $h$. The boundaries are all continuous as in the purely hydrodynamical case (Terra-Homem \& Erdélyi 2003). In this case, however, there are two more free variables which are the horizontal $\left(B_{x}\right)$ and vertical $\left(B_{y}\right)$ components of the magnetic field. The vertical component is always taken to be zero and the horizontal component may be uniform or varying from one side to the other of the shear layer. These two distinct cases are presented below in more detail.

The problem of initially perturbed open shear flows is solved numerically using the program VAC (Versatile Advection Code). A detailed description of VAC can be found in e.g., Tóth (1996). The Flux Corrected Transport (FCT) method is used to carry out the fully non-linear $2 \mathrm{D}$ simulations in a $400 \times 800$ uniform mesh.

In order to keep $\nabla \cdot \boldsymbol{B}=0$ a projection scheme was used. The projection scheme uses a Poisson solver to limit the divergence of $\boldsymbol{B}$ to a maximum stipulated value. For more details on the projection scheme and the $\nabla \cdot \boldsymbol{B}=0$ condition see Tóth (2000). In the simulations carried out for this study the maximum value allowed for $\nabla \cdot \boldsymbol{B}$ was $10^{-6}$.

\subsection{Uniform magnetic field}

In the case of a uniform magnetic field, $B_{x}$ is a constant, the equilibrium can be represented as shown in Fig. 1.

The magnetic field is parallel to the velocity shear layer, and the density is constant in the whole domain. The density is also unity because it is assumed that the density is scaled by a characteristic density $\rho_{0}$. The strength of the magnetic field is measured by the plasma $\beta$ parameter which is defined as follows

$\beta=\frac{2 c_{\mathrm{s}}^{2}}{\gamma_{\mathrm{ad}} v_{\mathrm{A}}^{2}}$,

where $v_{\mathrm{A}}$ is the Alfvén speed

$v_{\mathrm{A}}=\sqrt{\frac{B^{2}}{\rho}}$. 


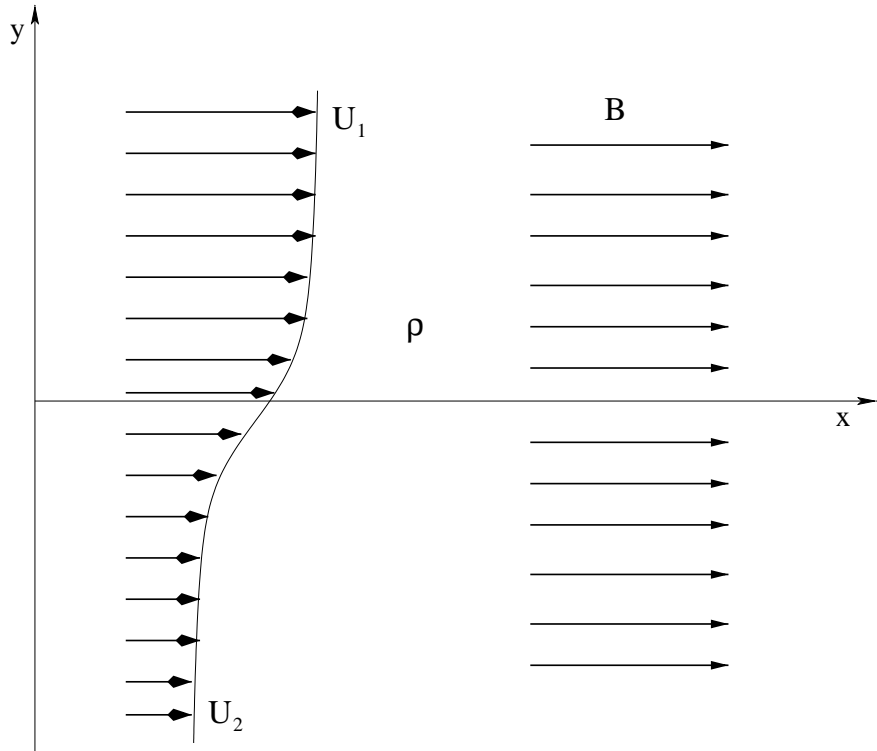

Fig. 1. Cartoon of the equilibrium in Cartesian coordinates for uniform magnetic field.

Once the value of $\beta$ is given $B_{x}$ can be calculated as

$B_{x}=\sqrt{\frac{2 c_{\mathrm{a}} \rho^{\gamma_{\mathrm{ad}}}}{\beta}}$.

The parameters for defining the equilibrium are then the mean velocity $\left(U_{0}=\left(U_{1}+U_{2}\right) / 2\right)$, the plasma $\beta$ and the sound speed $\left(c_{\mathrm{s}}\right)$. The sound speed is defined by the parameter $c_{\mathrm{a}}$ that is varied in order to change the sonic Mach number, which reads as

$M_{\mathrm{S}}=\frac{\Delta U}{c_{\mathrm{S}}}$.

In this configuration, since the density is constant, the sound speed is also constant.

\subsection{Sheared magnetic field}

The second case of this study will focus on accounts for differences in the magnetic field on both sides of the shear layer, as shown in Fig. 2.

There is a uniform magnetic field at $+\infty$ that shall be referred to as $B_{1}$, and a uniform magnetic field at $-\infty$ designated as $B_{2}$. The transition of the magnetic field is defined by a smooth function (tanh), as in the case of the velocity transition, and reads as follows

$B_{x}=\bar{B}+B_{r} \tanh (2 y / L)$.

Here $L$ is the thickness of the magnetic shear layer. The average magnetic field $(\bar{B})$ and the ratio of magnetic fields $\left(B_{\mathrm{r}}\right)$ are defined as

$\bar{B}=\frac{B_{1}+B_{2}}{2}$,

$B_{\mathrm{r}}=\frac{B_{1}-B_{2}}{2}$.

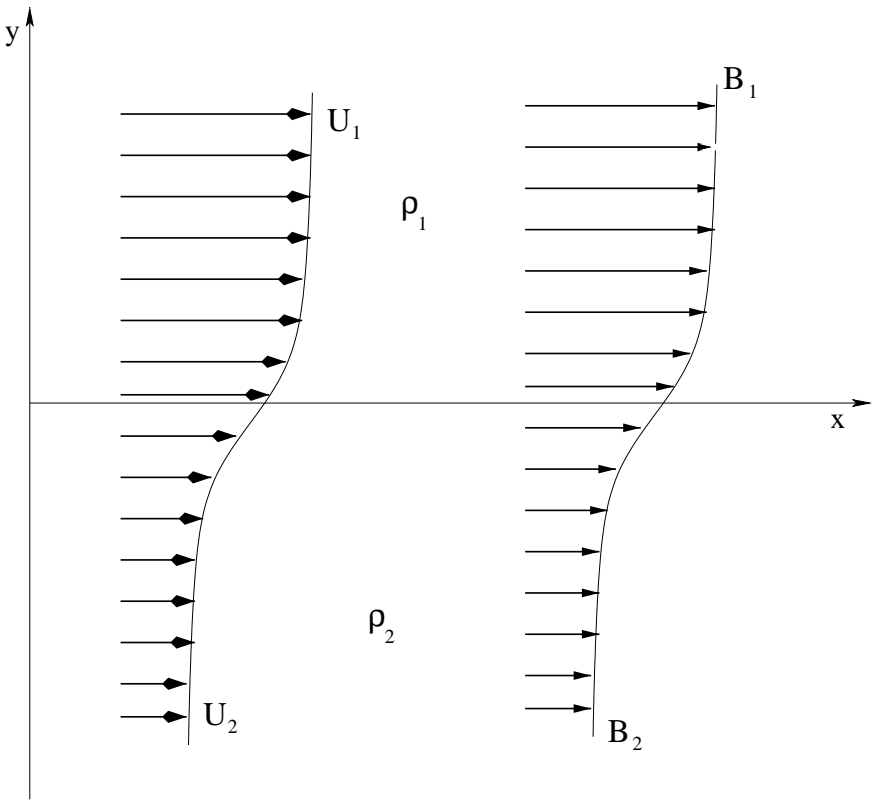

Fig. 2. Cartoon of the equilibrium in Cartesian coordinates for different magnetic fields on each side of the shear layer.

The strength of the magnetic fields is calculated from the plasma $\beta$ parameter using Eq. (7), and the ratio between the plasma beta at $+\infty\left(\beta_{1}\right)$ and the plasma beta at $-\infty\left(\beta_{2}\right)$. In this case $\rho_{1}$ is assumed to be unity and scaled by $\rho_{0}$, but the density can no longer be uniform in order to satisfy the condition of constant total pressure across the shear layer. The density profile must account for the change in the magnetic field and can be calculated using Eqs. (4) and (5). The ratio $\rho_{1} / \rho_{2}$ is obtained from the ratio $\beta_{1} / \beta_{2}$. This means that the sound speed is no longer constant as in the previous case, although the change is very small since the ratio $\rho^{\gamma_{\text {ad }}} / \rho$ is very close to unity throughout the whole domain.

In order to define the equilibrium configuration the parameters used as input are the same as in the previous section, but now there are also two plasma $\beta$ variables $\left(\beta_{1} ; \beta_{2}\right)$ and the size of the magnetic shear layer $(L)$. Note that the size of the magnetic shear layer does not necessarily have to be the same as the size of the velocity shear layer (i.e. $h \neq L$ ).

\section{Results and discussion}

\subsection{Kelvin-Helmholtz stability threshold with uniform magnetic field}

In the case of a velocity discontinuity it is possible to derive analytical solutions to the stability problem. The instability due to this velocity discontinuity is the well known Kelvin-Helmholtz instability. When there is a uniform magnetic field present this instability can be suppressed if the magnetic field intensity is strong enough. The condition for the magnetic field intensity required to stabilise a velocity discontinuity in a perfectly conducting, inviscid, compressible fluid with uniform density and uniform magnetic field was derived by Fejér (1964). 


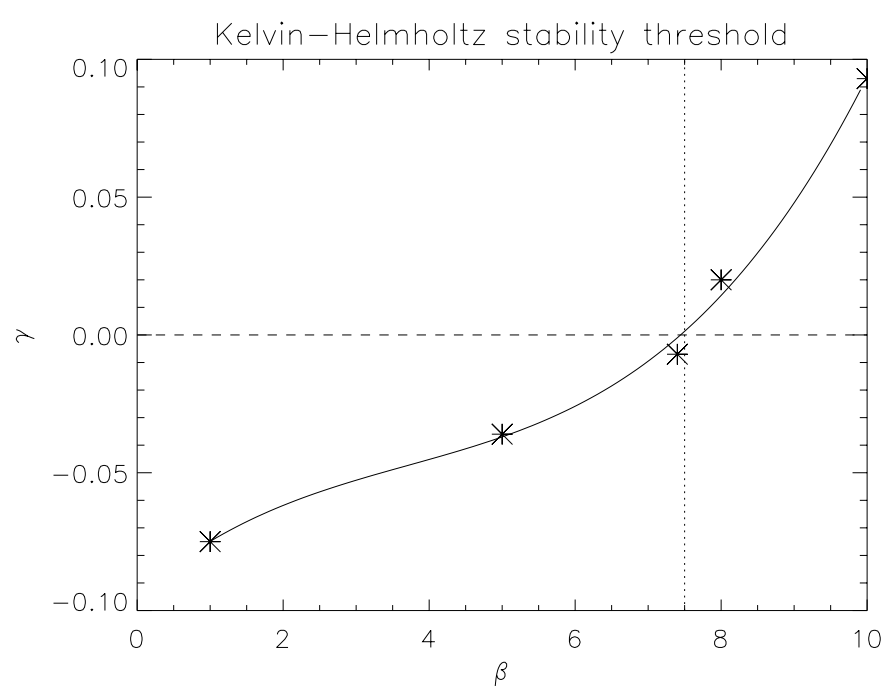

Fig. 3. Growth rate variation with plasma $\beta$ for a velocity discontinuity with uniform magnetic field $\left(M_{\mathrm{s}}=0.83 ; U_{0}=0\right)$. The vertical dotted line indicates the threshold value for which the interface becomes unstable.

This condition can be modified in order to give the condition for $\beta$ to stabilise a velocity discontinuity, and can be written as

$\beta<\left(\frac{2 \sqrt{2} c_{\mathrm{s}}}{\sqrt{\gamma_{\mathrm{ad}}} \Delta U\left(1+\frac{\Delta U^{2}}{16 c_{\mathrm{s}}^{2}}\right)}\right)^{2}=\beta_{\mathrm{c}}$.

Note, the smaller the value of $\beta$ the larger the intensity of the magnetic field, since the sound speed is fixed. Substituting the values in Eq. (10) for the ones used in the simulations $\left(c_{\mathrm{s}}=\right.$ $\left.1.28 ; \Delta U=1 ; \gamma_{\mathrm{ad}}=1.66\right)$, it is found that for values of $\beta<$ 7.45 the velocity discontinuity is stable.

Simulations are carried out for different values of $\beta$ and the growth rate is calculated. The results are shown in Fig. 3. Note that a velocity discontinuity can be considered as a velocity shear layer with width $h=0$. In reality the width of the shear layer is not zero and the transition is smoothed over a small number of grid cells. The real value of $h$ is 0.1 for simulating a discontinuity, as can be seen in Table 1.

The stability condition derived from the numerical results is found to be that for $\beta<7.5$ the velocity discontinuity interface is stable. Figure 3 shows that the growth rate crossing from a positive value to a negative value occurs at $\beta=7.5$, as can be seen by the dotted line. This is in excellent agreement with the analytical result of 7.45 . The difference, of only $0.6 \%$ in the threshold found, is due to the error introduced when calculating the growth rate $(\gamma)$.

In Fig. 4 the streamlines of a discontinuous velocity profile with a uniform magnetic field are plotted where $\beta=10$, $M_{\mathrm{s}}=0.83$ and $U_{0}=0$. Figure 4 clearly shows the interface has become unstable and it destroys the original equilibrium. It can be also observed that the magnetic field lines follow the streamlines of the fluid, as expected, since there is no magnetic resistivity and so the frozen in condition must be satisfied.

The magnetic field lines are shown to be distorted by the flow, and, because of the magnetic force acting against the

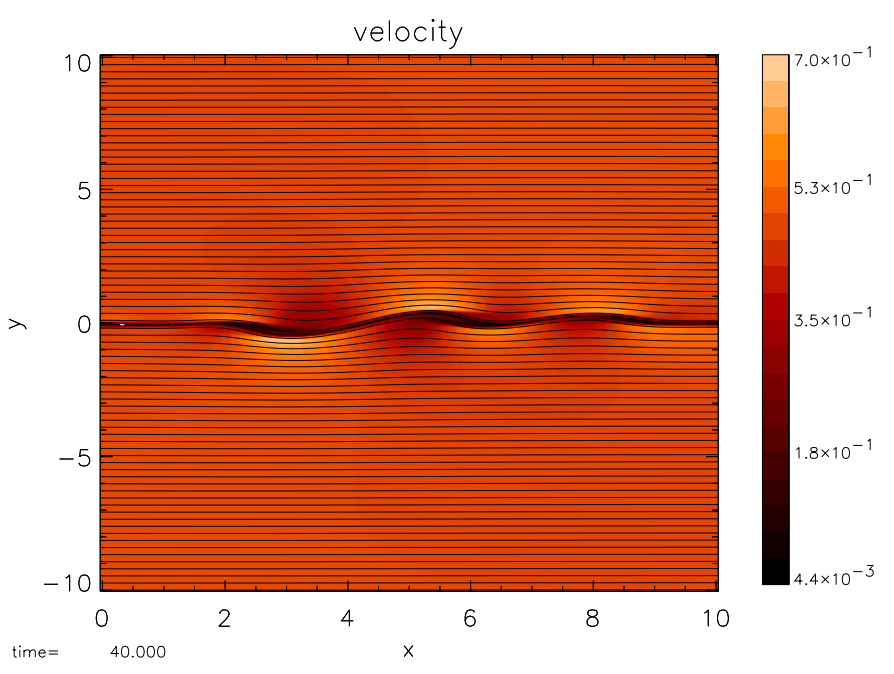

Fig. 4. Streamlines of $|\boldsymbol{v}|$ in a discontinuous velocity profile with uniform magnetic field, for the whole domain of computation for $\beta=10$, $M_{\mathrm{s}}=0.83$, and mean velocity $U_{0}=0$. The colour scale indicates intensity of velocity in dimensionless units, and the contour lines are the vector potential of the magnetic field.

curvature of the field, there is a restoring force that counteracts the instability. If that restoring force is strong enough then the instability is suppressed and the interface becomes stable. This is the physical reason for which a uniform magnetic field can stabilise the velocity discontinuity. That is also the reason why a transverse magnetic field will not have much influence on the Kelvin-Helmholtz instability (see Chandrasekhar 1961, Chap. XI).

\subsection{Case of continuous velocity transition and uniform magnetic field}

If, instead of having a tangential discontinuity in the flow velocity, we introduce a continuous change of the velocity profile, defining a shear layer with a finite thickness. Chandrasekhar (1961) found for the hydrodynamic case, and Ong \& Roderick (1972) for the hydromagnetic case, that a finite boundary thickness has a quenching effect on the instability for large wavenumbers. It is to expect then that the introduction of a shear layer will result in a more stable configuration than the one in Sect. 3.1. This effect was also observed in the numerical results by Terra-Homem \& Erdélyi (2003) for a shear flow layer. If the effect of the layer of finite thickness is to stabilise the flow, than it can be predicted that a smaller magnetic field, compared with the one in Sect. 3.1, is required to stabilise the flow. This results in a larger value for $\beta_{\mathrm{c}}$ since it is inversely proportional to the intensity of the magnetic field. Indeed this was observed by our numerical simulations, for which the critical value for the plasma beta is found to be $\beta_{\mathrm{c}}=8$ (see Fig. 5) for a hydromagnetic shear layer. The introduction of a finite thickness boundary layer increases the threshold value of $\beta_{\mathrm{c}}$ by $6 \%$. The plot in Fig. 5 shows that the growth rate decreases with decreasing plasma $\beta$. This is expected because as the value of the magnetic field intensity is increased, i.e. decreasing $\beta$, it will cause the magnetic forces to become dominant over the 


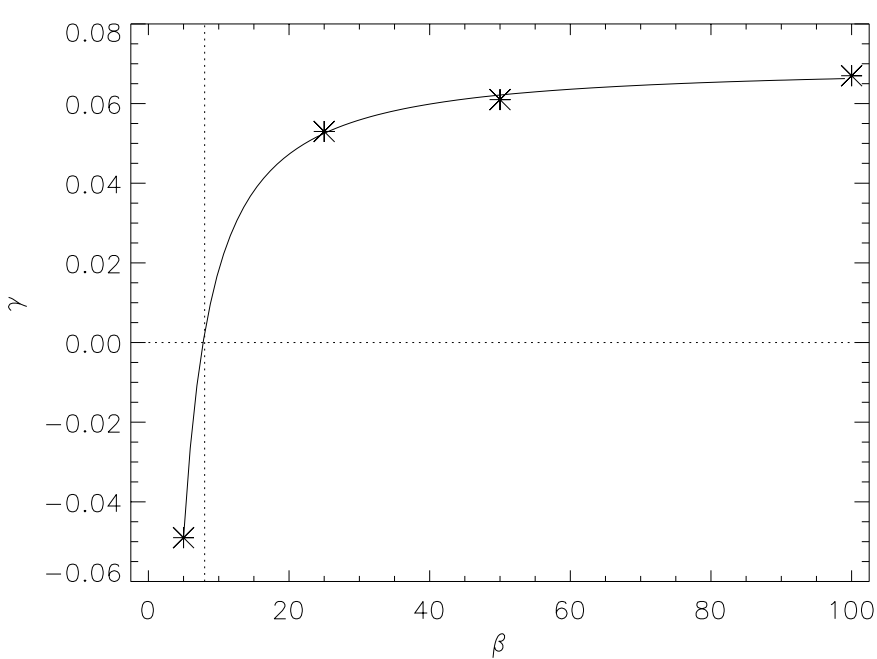

Fig. 5. Growth rate variation with plasma $\beta$ for a velocity shear layer with uniform magnetic field $\left(M_{\mathrm{s}}=0.83 ; U_{0}=0\right)$. The vertical dotted line indicates the threshold value for which the interface becomes unstable.

instability. The unexpected result is, however, that this transition is quite abrupt. The growth rate remains almost constant for values of $\beta$ ranging from 100-40 and then drops very quickly in the range of 30-5. This abrupt transition is in fact an artifact of the scale used. The sharp transition is caused by plotting the growth rate $(\gamma)$ to the plasma beta $(\beta)$. If instead the inverse of the plasma beta is used $\left(1 / \beta \propto v_{\mathrm{A}}\right)$, the abrupt transition is eliminated, as can be observed in Fig. 6 .

The condition of quenching large wavenumbers (i.e. small wavelengths), by the introduction of a finite thickness layer, can be noticed when comparing Fig. 7 with Fig. 4 . When the shear flow layer is present (Fig. 7) the instability has clearly a much larger wavelength than in the case of a discontinuity in the flow (Fig. 4). This increase in the wavelength might, at first instance, mean that the size of the domain in the $x$ direction must be increased. However, due to the following two points we hope to show that it is acceptable to maintain the size of the domain.

The first point is that continuous boundaries are used. Unlike periodic boundaries, that have a big influence on the growth rate, continuous boundaries have a small effect. On the other hand, the size of the domain will restrain the maximum wavelength we can study. That is, the size of the domain acts as a cut-off value for the wavelength and that has an effect on the instability. The next point will explain why this can be neglected.

One must consider what is the purpose of this study, and that is to find the influence of the magnetic field and its different configurations on the critical mean flow $\left(U_{\mathrm{c}}\right)$. It is not the purpose to do a detailed study of the instability itself. Having that in mind, there is only the need that the interface be unstable for $U_{0}=0$. When the value of $U_{0}$ is increased we show that the behaviour of the instability changes. Since the only parameter changed is $U_{0}$, it can be concluded that it is due to the change in the mean flow that the nature of the instability has changed. The effect of the boundary becomes then irrelevant in this case, because it is always the same in all cases.

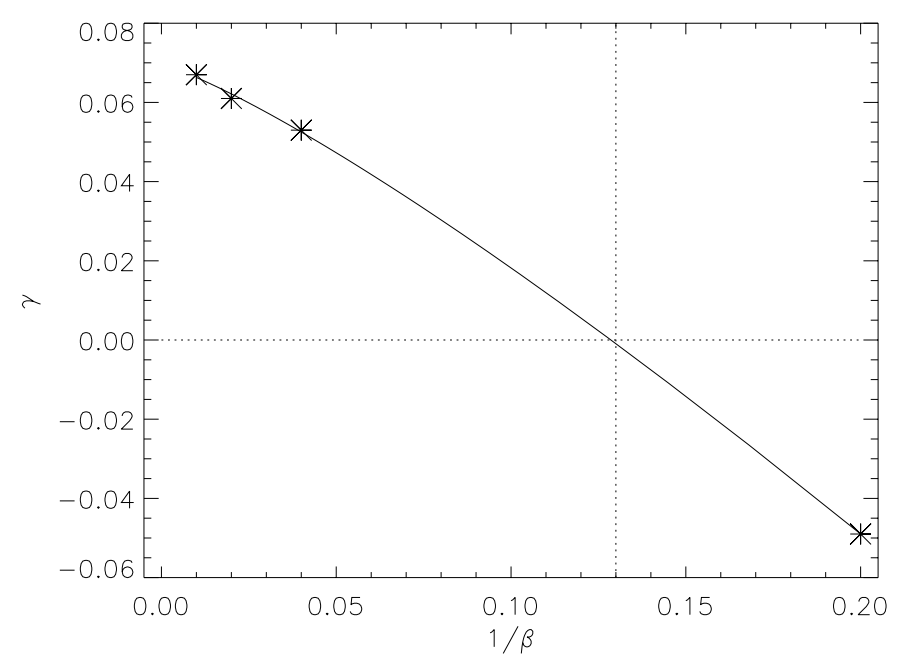

Fig. 6. Growth rate variation with $1 / \beta\left(\propto v_{\mathrm{A}}\right)$ for a velocity shear layer with uniform magnetic field $\left(M_{\mathrm{s}}=0.83 ; U_{0}=0\right)$. The vertical dotted line indicates the threshold value for which the interface becomes unstable.

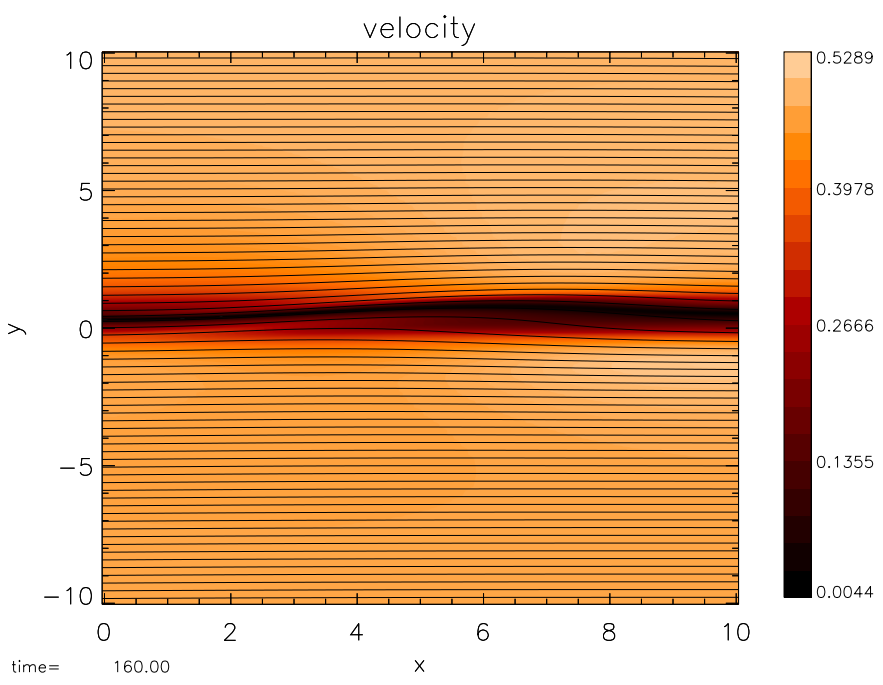

Fig. 7. Streamlines of $|\boldsymbol{v}|$ in a continuous velocity profile with uniform magnetic field, for the whole domain of computation for $\beta=25, M_{\mathrm{s}}=$ 0.83 , and mean velocity $U_{0}=0$. The colour scale indicates intensity of velocity in dimensionless units, and the contour lines are the vector potential of the magnetic field.

The only point where caution must be taken is when comparing the results for different values of $h$. This is however avoided in the present work, and the discontinuous case is present as a test case to corroborate our simulations. With the exception of Sect. 3.1, the value of $h$ is always the same (see Table 1). This also enables to maintain a continuity with the work done in Terra-Homem \& Erdélyi (2003).

As in the previous case there is a curvature in the magnetic field caused by the flow disturbance, which will cause the magnetic force to counteract this curvature stabilising the flow if the magnetic field is strong enough.

Due to this stabilising effect the growth of the instability will be reduced, since some energy is used to cancel the restoring force of the magnetic field. From a point of view of absolute and convective nature of the instability, this results in a smaller 


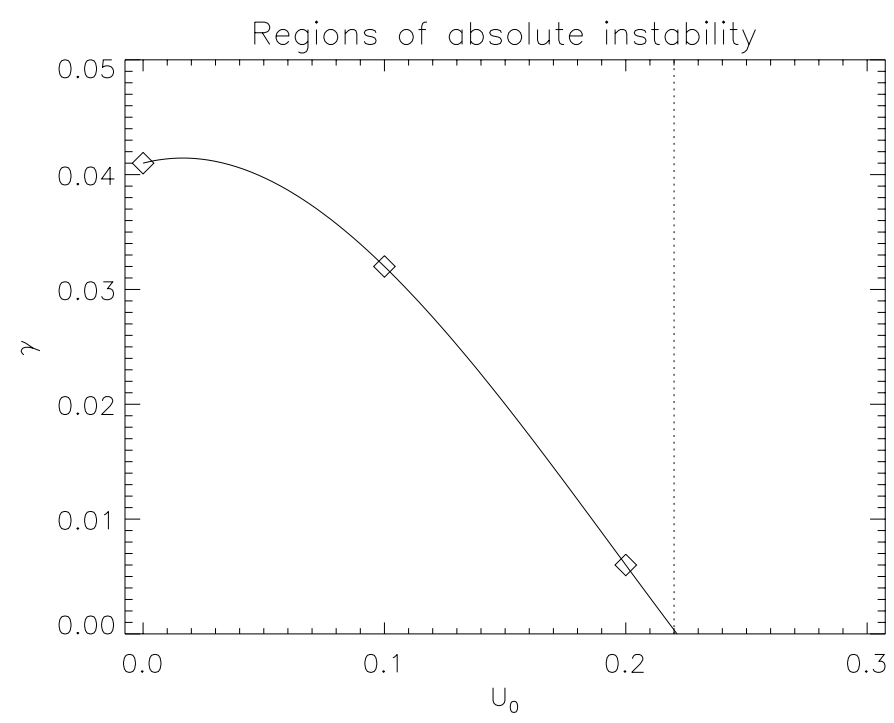

Fig. 8. Plot of growth rates for different values of mean flow $U_{0}$. The plasma parameter $\beta=20$ and $M_{\mathrm{s}}=0.83$. The vertical dotted line indicates the threshold value for which the instability becomes convective $\left(U_{\mathrm{c}}\right)$.

mean flow velocity needed to sweep away the perturbation before it grows everywhere. This translates to a smaller value of $U_{\mathrm{c}}$ and can be verified in Fig. 8 where the growth rates are plotted for different values of the mean flow $\left(U_{0}\right)$, with $\beta=20$.

From Fig. 8 it is found that the critical value of mean flow is $U_{\mathrm{c}}=0.22$. From this it is concluded that even a small magnetic field $(\beta=20)$ has a significant change in the value of $U_{\mathrm{c}}$. Comparing the value found in the case with no magnetic field, $U_{\mathrm{c}}=0.37$ (see Terra-Homem \& Erdélyi 2003), with the value of 0.22 , here there is an $\approx 40 \%$ reduction in the critical value of mean flow.

If the shear is increased one would expect that the instability would be stronger and so a bigger magnetic field would be needed in order to stabilise the flow. However, this is quite a simplistic picture, and, as shall be demonstrated next the flow can actually be stable for large values of shear. Recall that this study only considers perturbations travelling parallel to the flow, and this turns out to be important and critical when explaining the stabilisation observed in the simulations. To illustrate this, two conditions for stability (or instability) must be considered.

The first condition is what will be referred to as the acoustic condition of instability. For values of sonic Mach number (Eq. (8)) larger than $2 \sqrt{2}$, Landau (1944) determined that the shear flow becomes stable. When the flow velocity becomes so large that the wave can no longer interact with the flow to create the instability, this results in a stable configuration (see Landau 1944; Baranov et al. 1992). This condition is only valid for perturbations parallel to the flow. In the general case there is always a direction in which the perturbation is unstable and as the Mach number increases the cone of propagation for the unstable modes is reduced (Syrovatskii 1954).

On the other hand there is the magnetic field which can stabilise the flow; this second condition will be referred to as

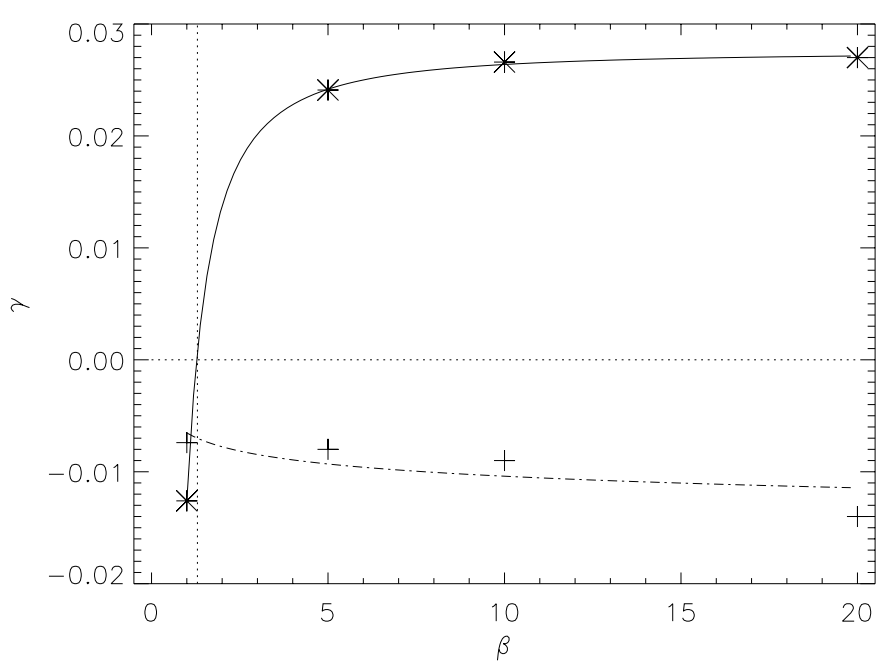

Fig. 9. Growth rate variation with plasma $\beta$ for a velocity shear layer with uniform magnetic field $\left(U_{0}=0\right)$, for $M_{\mathrm{s}}=1.66$ (full line) and $M_{\mathrm{s}}=4.15$ (dash-dotted line). The vertical dotted line indicates the threshold value for which the interface becomes unstable.

the magnetic condition. A new Mach number is defined, called the Alfvénic Mach number, which can be written as

$M_{\mathrm{A}}=\frac{\Delta U}{v_{\mathrm{A}}}$,

where $v_{\mathrm{A}}$ is the Alfvén speed. In this case the condition for stability is the Alfvénic Mach number to be smaller than unity (see Ruderman \& Wright 1998). This can be easily understood: $v_{\mathrm{A}}$ is proportional to the intensity of the magnetic field, so for low values of $M_{\mathrm{A}}, v_{\mathrm{A}}$ is large and the magnetic field is strong enough to stabilise the flow. For $M_{\mathrm{A}}>1, v_{\mathrm{A}}$ is small compared with the shear and the magnetic field can no longer stabilise the flow resulting in an unstable configuration.

By inspecting these two conditions carefully one can immediately conclude that they are in fact counteracting each other. The first condition (acoustic) requires small values of shear, while the second (magnetic) requires large values of shear. Therefore increasing the value of the shear at first will cause the flow to be more unstable, due to the magnetic condition. If the value is further increased to a value so that the acoustic condition is achieved the flow will actually stabilise. Figure 9 shows that the critical $\beta$ decreases, $\beta_{\mathrm{c}}=1.3$, for a sonic Mach number that is doubled which is equivalent to doubling the amount of shear $\left(M_{\mathrm{s}}=1.66\right)$. For $M_{\mathrm{s}}=4.15$ the flow is actually stable for all of the $\beta$ values as shown in Fig. 9.

Another point to make is that due to this conflicting conditions low $\beta$ plasmas, or so called cold plasmas, will always be stable to perturbations propagating parallel to the flow (Ruderman \& Goossens 1996). Recalling that $\beta$ is a measure of relative strength of acoustic and magnetic forces, the case of $\beta=1$ means that $c_{\mathrm{s}}$ and $v_{\mathrm{A}}$ have a ratio of $\gamma_{\mathrm{ad}} / 2 \approx 1$ (see Eq. (6)) which means that $M_{\mathrm{s}} \approx M_{\mathrm{A}}$. If these two characteristic speeds are about the same value, this will give a very small window for the amount of shear that can satisfy both conditions for instability. In fact it can be estimated that only values of $M_{\mathrm{s}}$ between 1 and $2 \sqrt{2}$ can lead to an unstable flow for the 
$\beta=1$ case. However the general picture is a lot more stable. This is because the conditions derived by Landau (1944) are for an interface of discontinuous velocity, and in the present section there is a continuous transition of velocity that has been proved to stabilise the flow. Therefore the window for instability is much smaller. Simulations were carried out for cases of $\beta=1$ with $M_{\mathrm{s}}=0.83, M_{\mathrm{s}}=1.17, M_{\mathrm{s}}=1.66$ and $M_{\mathrm{s}}=2.34$ where all of this cases were found to be stable. If the value of $\beta$ is decreased the window for instability decreases and at some point it vanishes by the flow becoming stable for all values of $M_{\mathrm{s}}$.

\subsection{Case of continuous velocity transition and sheared magnetic field}

In this section we study the effect of a magnetic shear layer on the stability of shear flows. The magnetic field is changing from one side of the shear layer to the other. The change of the magnetic field can occur in two different ways: it is discontinuous, i.e. the transition in field intensity is sudden and occurs at $x=0$ (centre of the shear layer); it is continuous, i.e. the transition is a smooth function and is centered at $x=0$. In what follows we treat the two different cases separately. The discontinuous case of the magnetic field is not actually discontinuous, but an approximation to it. In this case $L=0.01$ (see Table 1), which actually covers only two grid cells. This is a very good approximation to a discontinuity.

\subsubsection{Discontinuous transition of magnetic field}

In Sect. 3.2 it was shown that for $M_{\mathrm{s}}=0.83$ the flow was stable for values of $\beta<8$, and unstable otherwise. The threshold value for the mean flow $U_{\mathrm{c}}$ was found to be 0.22 for the same sonic Mach number and $\beta=20$ (see Fig. 8). Therefore the first case to be studied has $M_{\mathrm{s}}=0.83, \beta_{1}=20$ and $\beta_{2}=8$, following the definitions shown in Fig. 2. According to the previous results this flow must be unstable since both plasma betas are above $\beta_{\mathrm{c}}$, and $U_{\mathrm{c}}$ can therefore be calculated and compared with the one where the plasma $\beta$ is 20 everywhere. The results from the numerical simulations for this case are shown in Fig. 10, and it can be seen that $U_{\mathrm{c}}=0.075$.

Comparing this value $\left(U_{\mathrm{c}}=0.075\right)$ with the value found when the entire plasma is uniform $(\beta=20)$ we found $U_{\mathrm{c}}=$ 0.22 . A difference of more than half is observed! This difference appears because the plasma $\beta$ on side 2 is at the edge of the value for which it can stabilise the flow. Another few simulations were carried out for cases where all the parameters were the same with the exception of the value of $\beta_{2}$, which was reduced to e.g. 6 and 2. In both cases the flow was stable although the plasma $\beta$ on side 1 was above the critical value and the plasma should be unstable. This suggests that the predominant behaviour is governed by the lower $\beta$ plasma, and if one of the sides has a plasma $\beta$ below the critical value then the entire interface will be stable.

We note, that the unstable side does have an effect that can be observed in the value for the threshold of the mean flow for which the shear flow becomes convectively unstable. In Fig. 10

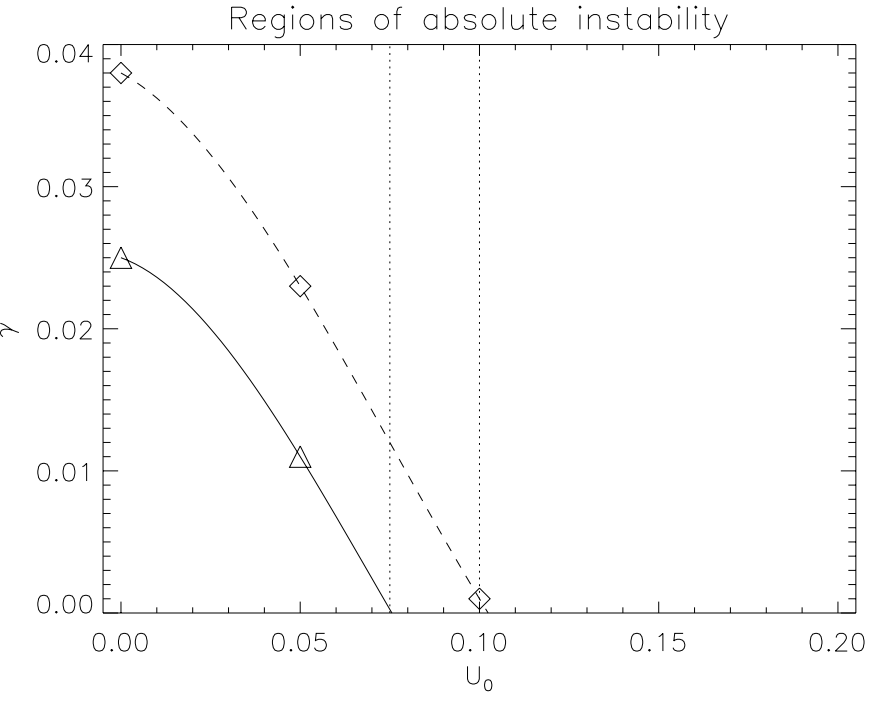

Fig. 10. Plot of growth rates for different values of mean flow $U_{0}$ with $M_{\mathrm{s}}=0.83$. The full line has $\beta_{1}=20 ; \beta_{2}=8$ and the dashed line has $\beta_{1}=40 ; \beta_{2}=8$. The vertical dotted lines indicate the threshold values for which the instability becomes convective $\left(U_{\mathrm{c}}\right)$.

it can be seen that for $\beta_{1}=40$, which is double the value of $\beta$, the value of $U_{\mathrm{c}}$ is changed by $25 \%$. This indicates that the larger the plasma $\beta$ on one side the more flow is needed to sweep away the perturbation, which is in agreement with the observation that the magnetic field intensity is lower in that case and so the instability is stronger and grows more quickly.

\subsubsection{Continuous transition of magnetic field}

Let us now consider a finite length over which the magnetic field changes from $B_{1}$ to $B_{2}$. A new variable is introduced $h / L$, where $L$ is the thickness of the magnetic shear layer and $h$ the thickness of the velocity shear layer. Therefore the case of discontinuous transition of the magnetic field, discussed previously, can be considered here as $h / L \rightarrow \infty$. Representative simulations are shown here for two different widths of the magnetic shear layer and are compared with the discontinuous case for $M_{\mathrm{s}}=0.83$ with $\beta_{1}=20$ and $\beta_{2}=10$. The results are plotted in Fig. 11.

It is observed from the numerical results that changing the width of the transition layer for the magnetic field has no significant change in the value of $U_{\mathrm{c}}$. The differences in the value of $U_{\mathrm{c}}$ observed in Fig. 11 are caused by numerical errors when trying to fit the exponential curve to the numerical data. The maximum difference is only 0.01 . This is even more assured by inspecting that the value of $U_{\mathrm{c}}$ for the discontinuous case is larger than for $h / L=1$ and smaller than for $h / L=0.5$, showing no trend of behaviour with decreasing $h / L$. The critical value of mean flow can then be averaged from the three values found, which results in $U_{\mathrm{c}}=0.18$.

\section{Conclusions}

In this paper we studied the effect of magnetic field in the value of critical mean flow necessary for an instability to become 


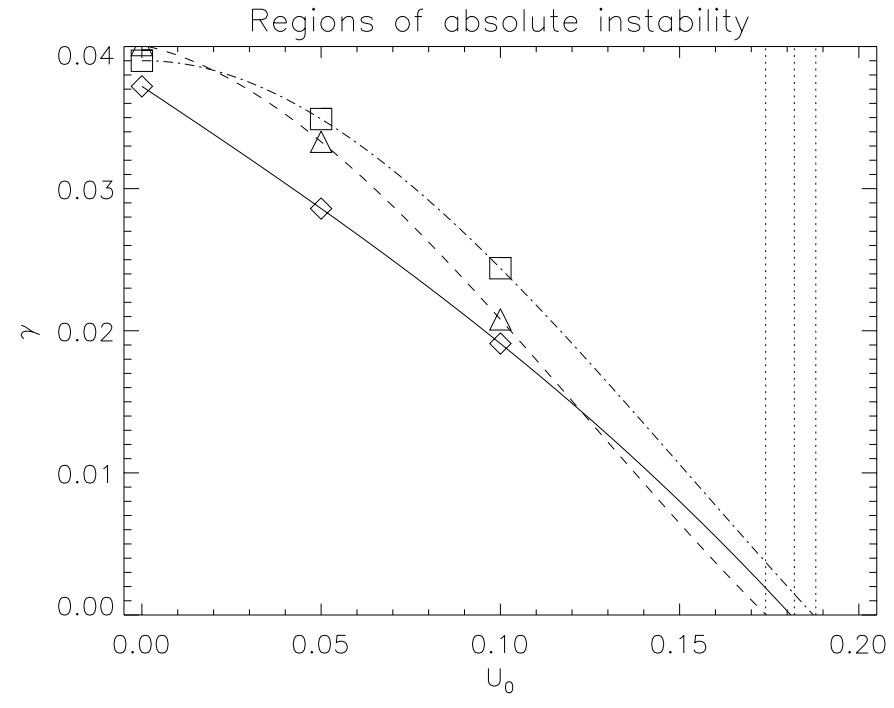

Fig. 11. Plots of growth rates for different values of mean flow $U_{0}$. The plasma parameters are $\beta_{1}=20, \beta_{2}=10$ and $M_{\mathrm{s}}=0.83$. The full line represents $h / L \rightarrow \infty$, the dashed line $h / L=1$ and the dash-dot line $h / L=0.5$. The vertical dotted lines indicate the threshold values for which the instability becomes convective $\left(U_{\mathrm{c}}\right)$.

convectively unstable. Three different cases were studied: (i) an uniform magnetic field; (ii) a discontinuous magnetic field; (iii) and a magnetic field with a smooth transition.

The numerical simulations carried out here are able to recover the analytical results for a discontinuous velocity transition of the intensity needed for the magnetic field in order to stabilise the interface. These served as test cases.

The inclusion of a finite thickness transition layer for the velocity resulted in a more stable configuration than the discontinuous transition. Less magnetic field intensity was found to stabilise the interface in the case where the transition of velocity was a smooth function. This conclusion has to be taken carefully, because there is the problem of the size of the domain. In this case it may be important to consider that the size of the domain may in fact be stabilizing the interface and not the inclusion of the shear layer. We suspect that it is in fact a combination of both. It was also found that even if the magnetic field is not sufficient to stabilise the interface the magnetic field still has a strong effect on the behaviour of the instability. The necessary mean flow to sweep away growing perturbations was found to decrease by $40 \%$ even for a weak magnetic field $(\beta=20)$. This could be explained because the magnetic field hinders the rate of growth of the perturbation, due to magnetic tension and restoring forces.

When different magnetic fields were allowed on the two different sides of the shear layer it was found that, if the plasma $\beta$ on one of the sides was low enough to stabilise the interface in the case of a uniform magnetic field, then the interface would be stable. This result agrees with another result which is that the transition from unstable to stable due to the reduction of $\beta$ is quite abrupt. So, as soon as that critical value is reached the side with low $\beta$ plasma will cancel out the effect of the side with high $\beta$ plasma. This behaviour can be better understood by inspecting Fig. 5 where the plot has almost a behaviour of a step function.

When both of the sides have $\beta$ values above the critical value then the interface is unstable. In this case there is a direct effect of the values of plasma $\beta$ on each side on the threshold value for the mean flow $\left(U_{\mathrm{c}}\right)$. It was found that the higher the value of $\beta$ on one side the higher the value of $U_{\mathrm{c}}$, which means that more flow is needed in order for the perturbation to become convectively unstable. Once again this confirms that for high $\beta$ values the magnetic field intensity decreases and so the magnetic forces are weaker and cannot counteract the instability as much as in the case of a lower value of $\beta$. The inclusion of a finite thickness transition layer for the magnetic field proved to have no effect on the value of the critical mean velocity, $U_{\mathrm{c}}$, for all the three different lengths of the layer.

Finally we wish to make some remarks. First it is important to note that this study is for parallel propagation only, which has significant constraints. It would be interesting to extend the study for non-parallel propagation were the interface would still be unstable even for low $\beta$ values. Such study is very important because many astrophysical problems deal with cold plasmas, as a first approximation. One example is the cavity of the magnetosphere. Second, we found that the thickness of the magnetic shear layer has practically no effect on the nature of the instability. This may be because this study used the limit of ideal MHD approximation. The inclusion of a dissipative term may lead to the presence of resonant instability that is effected by the thickness of the magnetic shear layer (see the recent analytical study by Erdélyi \& Taroyan 2003). The critical point here is that the threshold of resonant flow instability was found below the KHI threshold in various normal mode analysis. To the best of our knowledge there are no studies of the absolute and convective nature of resonant flow instabilities. It would also be most interesting to try and recover numerically in a forward problem the results found by the normal mode analysis of Erdélyi \& Taroyan (2003).

Acknowledgements. MTH thanks FCT, Portugal, for its financial support (Ref. No. SFRH/BD/8076/2002). RE acknowledges M. Kéray for patient encouragement and is also grateful to NSF Hungary, (OTKA, Ref. No. TO43741). We also acknowledge Dr M. S. Ruderman for the many useful discussions and comments and G. Tóth the principal author of VAC.

\section{References}

Axford, W. I. 1960, Quart. J. Mech. Appl. Math., 13, 314

Axford, W. I. 1962, Can. J. Phys., 40, 654

Baranov, V. B., Hans, J., \& Ruderman, M. S. 1992, A\&A, 261, 341

Chandrasekhar, S. 1961, Hydrodynamic and Hydromagnetic Stability (Oxford University Press)

Erdélyi, R., \& Taroyan, Y. 2003, J. Geophys. Res., 108, doi: 10.1029/2002JA009586

Fejér, J. A. 1964, Physics Fluids, 7, 499

Landau, L. D. 1944, Dokl. Akad. Nauk SSSR, 44, 151

Mills, K. J., Longbottom, A. W., Wright, A. N., \& Ruderman, M. S. 2000, J. Geophys. Res., 105, 27685 
Ong, R. S., \& Roderick, N. 1972, Planet. Space Sci., 20, 1

Ruderman, M. S. 2000, Ap\&SS, 274, 327

Ruderman, M. S., Brevdo, L., \& Erdélyi, R. 2002, Proc of Royal Soc. Lond., submitted

Ruderman, M. S., \& Fahr, H. J. 1993, A\&A, 275, 635

Ruderman, M. S., \& Fahr, H. J. 1995, A\&A, 299, 258

Ruderman, M. S., \& Goossens, M. 1996, ApJ, 471, 1015

Ruderman, M. S., Verwichte, E., Erdélyi, R., \& Goossens, M. 1996, J. Plasma Phys., 56, 285
Ruderman, M. S., \& Wright, A. N. 1998, J. Geophys. Res., 103, 26573 Syrovatskii, S. I. 1954, J. Exp. Teor. Phys., 27, 121

Terra-Homem, M., \& Erdélyi, R. 2003, A\&A, 403, 425

Tóth, G. 1996, Astrophys. Lett. Comm., 34, 245

Tóth, G. 2000, J. Comp. Phys., 161, 605

Wright, A. N., Longbottom, A. W., Mills, K. J., \& Ruderman, M. S. 2002, J. Geophys. Res., 107, 14

Wright, A. N., Mills, K. J., Ruderman, M. S., \& Brevdo, L. 2000, J. Geophys. Res., 105, 385 